\title{
Algorithms and models for complex natural systems
}

\author{
Carlos A. Coello Coello ${ }^{1}$ - Giuditta Franco ${ }^{2}$ - Natalio Krasnogor ${ }^{3}$. \\ Mario Pavone ${ }^{4}$
}

Published online: 7 May 2015

(C) Springer Science+Business Media Dordrecht 2015

Analysing genomic data and complex natural phenomena in computational terms enhances our comprehension of both nature and computation. Thus, a cross fertilization of algorithms and models for natural complex systems at molecular, cellular, or higher levels, became an active research area, and a more in depth investigation of mutual relationships, synergies, similarities, and differences should be encouraged.

This special issue is meant to foster novel hybrid approaches, including general methods of (bio) informatics and synthetic biology, as well as to present the new emerging research concerned with the study and analysis of genome organization, and with the design, modelling, and implementation of bio-inspired evolvable systems. Of particular interest are (unconventional) computational techniques designed to increase our understanding of the

Mario Pavone

mpavone@dmi.unict.it

Carlos A. Coello Coello

ccoello@cs.cinvestav.mx

Giuditta Franco

giuditta.franco@univr.it

Natalio Krasnogor

natalio.krasnogor@ncl.ac.uk

1 Depto. de Computación, CINVESTAV-IPN, Av. Instituto Politécnico Nacional No. 2508, Col. San Pedro Zacatenco, 07300 Mexico, D.F., Mexico

2 Department of Computer Science, University of Verona, Strada Le Grazie 15, 37134 Verona, Italy

3 School of Computing Science, Newcastle University, Claremont Tower, Newcastle NE1 7RU, UK

4 Department of Mathematics and Computer Science, University of Catania, Viale A. Doria 6, 95125 Catania, Italy evolution of biological life, such as algorithms to infer gene structure and functioning, and parallel distributed computational models involving mechanisms of recognition, affinity based discrimination, reactivity and adaptation to the environment, and spatial search and moving.

After a peer review process, 6 manuscripts were accepted for inclusion in this special issue.

In "Combining flux balance analysis and model checking for metabolic network validation and analysis", by Roberto Pagliarini, Mara Sangiovanni, Adriano Peron, and Diego Di Bernardo, the authors present a novel useful approach for extracting relevant qualitative information from a metabolic network model, integrating constraintbased techniques with model checking methods. This new computational approach may be helpful in understanding the mechanisms governing the onset and progression of human metabolic-related disorders. It was applied to a simulation and analysis of a well-known inherited disease (primary hyperoxaluria type I) where the lack of a particular liver enzyme causes the body to accumulate excessive amounts of oxalate, leading to renal failure.

In A hybrid method for inversion of $3 D D C$ resistivity login measurements, by Ewa Gajda-Zagórska, Robert Schaefer, Maciej Smołka, Maciej Paszyński, and David Pardo, the authors present a new hybrid method for solving the challenging inversion of 3D direct current (DC) resistivity logging measurements. This methodology consists of a $h p$ hierarchic genetic strategy ( $h p$-HGS), and a gradient based optimization method for a local search. The problem has been formulated as a global optimization problem, and simulations have been performed using a self-adaptive $h p$ finite element method. The experimental results demonstrate the suitability of the proposed method for the tackled inversion problem.

In An evolutionary procedure for inferring MP systems regulation functions of biological networks, by Alberto 
Castellini, Vincenzo Manca, and Mauro Zucchelli, the authors present a Metabolic P system (MP systems) for inferring knowledge of the regulatory mechanism of biochemical processes directly from observed dynamics. In particular, they introduce some important improvements based on the combination of genetic algorithms and multiple linear regressions. Three case studies have been conducted to perform an accurate analysis, which demonstrates that such methodology is able to infer significant knowledge of the regulation mechanisms underlying biological processes.

In Bayesian versus data driven model selection for microarray data, by Raffaele Giancarlo, Giosué Lo Bosco, and Filippo Utro, the authors present a study of a model selection methodologies in the contest of microarray data clustering. In particular, they have compared 9 data-driven model selection methodologies with 3 Bayesians methodologies (BIC, AIC, and MML). The experiments have been performed on 11 different benchmark microarray datasets. The obtained results show that, although the Bayesian methods guarantee good results, they cannot compete with the data-driven methods in terms of precision and ability to predict the correct number of clusters.

In A genome analysis based on repeat sharing gene networks, by Alberto Castellini, Giuditta Franco, and Alessio Milanese, the authors present new types of networks based on repeats in common between two genes for genomic sequence analysis. The study of these networks has been conducted on three organisms, each with different length of the common repeat, and it took into account both topological and biological perspectives. This paper demonstrates that (i) the cliques represent the structure with the greatest importance for both perspectives, and (ii) such networks provide an intuitive method to analyse and compare the three specific genomic sequences, where regularity phenomena have been found and characterized by repeat length range.

In Topology driven modelling: the IS metaphor, by Emanuela Merelli, Marco Pettini, and Mario Rasetti, the authors present a new topology-based method suitable for providing a benchmarking application of the $\mathrm{S}[\mathrm{B}]$ paradigm. The method relies on a multi-linear model of immune system inspired by the topology of space of data. The goal of this paper is to define a new approach to the analysis of the immune system as a metaphor for a real-life system represented in terms of Big Data.

The editors would like to thank to all authors who have submitted their novel and original manuscripts proving that the topic of this special issue is hot and challenging, as well as to the many reviewers who helped to ensure high quality of the manuscripts included in this special issue. We also want to thank the editors-in-chief of Natural Computing, Joost N. Kok, and Herman P. Spaink. Special thanks go to the founding editor-in-chief Grzegorz Rozenberg for encouraging and then accepting this special issue. 